\title{
An astronomical institute's perspective on meeting the challenges of the climate crisis
}

\author{
Analysing greenhouse gas emissions of an astronomical institute is a first step to reducing its environmental \\ impact. Here, we break down the emissions of the Max Planck Institute for Astronomy in Heidelberg and propose \\ measures for reductions. \\ Knud Jahnke, Christian Fendt, Morgan Fouesneau, Iskren Georgiev, Tom Herbst, Melanie Kaasinen, \\ Diana Kossakowski, Jan Rybizki, Martin Schlecker, Gregor Seidel, Thomas Henning, Laura Kreidberg and \\ Hans-Walter Rix
}

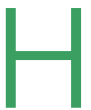
umanity's production of greenhouse gas (GHG) emissions is threatening our habitat, our physical and mental health, and the chances of long-term survival of human society as we know it ${ }^{1,2}$. The GHGs emitted as we burn fossil fuels for energy have already resulted in a mean surface temperature rise of more than $1{ }^{\circ} \mathrm{C}$ since the late nineteenth century ${ }^{3}$. To further limit the temperature rise to less than $1.5^{\circ} \mathrm{C}$ (as per the Paris Agreement ${ }^{4}$ ) requires all sections of human society to reduce their GHG emissions to net zero by 2050 . The scientific profession is not exempt. It is our responsibility to analyse the origin of our work-related emissions, to identify solutions for reducing emissions, and to determine the responsibility on a personal, institute-, community- and society-wide level for implementing the necessary changes.

As astronomers of the Max Planck Institute for Astronomy (MPIA) in Heidelberg, Germany, we have assessed our work-related GHG emissions. The MPIA is a well-funded, international astronomy research institute with $\sim 150$ researchers and 320 employees in total. A wide range of research is conducted at the institute, including the development of astronomical instrumentation, analysis of observational data, and theoretical modelling of astrophysical phenomena with computing facilities. The institute is scientifically well connected both within Europe and internationally, which, in combination with the broad range of research departments, makes it a good test case for the analysis of research-associated GHG emissions. This report can therefore serve as a template for other institutes. Our analysis provides a complementary, European perspective to the analysis on the Australian astronomical community $^{5}$, the Canada-France-

Hawaii Telescope ${ }^{6}$, the annual European Astronomical Society conferences ${ }^{7}$ and an earlier analysis of US astronomy ${ }^{8}$.

\section{MPIA GHG emissions}

We assessed the MPIA's GHG emissions in seven categories: business flights, commuting, electricity, heating, computer purchases, paper use, and cafeteria meat consumption. These categories were selected either because they were likely to have a large contribution or because we had no prior gauge of their significance. For this first assessment, we omitted other purchases, including materials and components for instrumentation, additional office supplies, and IT hardware other than desktop and laptop computers.

The GHG emissions associated with some categories were easily determined, for example from electricity and heating oil bills, computer expenses, paper purchases and recycling amounts. However, other categories proved less straightforward. Assessing the emission from flights required both a manual transcription of invoices and a questionnaire to all employees about self-booked business trips, as there was no automated and accessible list of itineraries, carriers or classes. Nevertheless, all the numbers quoted here (see Table 1 ) capture the MPIA's 2018 emissions quite well. We estimate the major contributors to our GHG emissions, that is, flying and electricity, to be accurate to within $20 \%$.

Table 1 summarizes the emission sources and the associated quantities. We have converted the units for each source into tons of $\mathrm{CO}_{2}$-equivalent emissions $\left(\mathrm{tCO}_{2} \mathrm{e}\right)$. The term 'equivalent' indicates that these values are normalized to the GHG impact of $\mathrm{CO}_{2}$. In particular, the numbers in this table account for flight emissions at altitude (for example, soot, sulfates, nitrogen oxides, and cirrus clouds from contrails), as well as methane emissions from meat farming.

The MPIA's total GHG emissions for 2018 amount to $18.1 \mathrm{tCO}_{2}$ e per researcher. Alternatively, the contribution per refereed science publication, of which there were
583 either authored or coauthored by MPIA astronomers in 2018 , is $4.6 \mathrm{tCO}_{2} \mathrm{e}$. However, regardless of the chosen denominator, these metrics have caveats in attribution. For example a substantial part of the institute's emissions results from instrumentation projects that will lead to future publications, but at the same time, we do not account for the emissions associated with the construction of observing facilities used in the 2018 papers; in addition, simulations can take months to years.

The MPIA's astronomy-related GHG emissions per researcher in 2018 were alarmingly around three times higher than the German target for 2030 (which is in line with the Paris Agreement; see Fig. 1) ${ }^{9-11}$. Moreover, the per-researcher emissions are $\sim 60 \%$ higher than those of the average German resident, whose annual 2018 GHG emissions (by consumption) were $11.6 \mathrm{tCO}_{2}$ e (refs. ${ }^{9,12,13}$; GHG emissions by consumption per adult resident were 14.0 $\mathrm{tCO}_{2} \mathrm{e}\left(\right.$ ref. $\left.\left.{ }^{12}\right)\right)$. Of course, these numbers just compare the work-related contributions of MPIA researchers to the Paris target and German averages, neglecting the additional emissions associated with non-research-related 'private' emissions by MPIA researchers, such as, for example, housing, clothing, private mobility, or food.

Few comparisons exist in the astronomical context. We therefore compare the MPIA's emissions to the recent assessment by the Australian astronomical community ${ }^{5}$. The MPIA's per-astronomer emissions are approximately half that of an Australian astronomer, which amount to $42 \mathrm{tCO}_{2}$ e per capita (see Fig. 1). Note that we calculated flight emissions using the model by atmosfair ${ }^{14}$, which estimates approximately double the emissions of the Qantas calculator ${ }^{15}$ used for the original Australian assessment ${ }^{5}$. Adjusting the reported Australian number by this factor, the MPIA's flight emissions are similar or 
Table 1 | Summary of the MPIA's GHG emissions in 2018

\begin{tabular}{lllll} 
Source & Amount & $\mathbf{t C O}_{\mathbf{2}} \mathbf{e}$ & tCO $_{\mathbf{2}} \mathbf{e}$ per researcher & Percentage (\%) \\
\hline Travel (air) & 1,030 flights & 1280 & 8.5 & 47 \\
\hline Electricity (on/off campus) & $3,400,000 \mathrm{kWh}$ & 779 & 5.2 & 29 \\
\hline Heating (oil) & 150,000 I & 446 & 3.0 & 16 \\
\hline Commuting (car) & $792,000 \mathrm{~km}$ & 139 & 0.9 & 5 \\
\hline Paper (cardboard) & $0.15(7) \mathrm{t}$ & 35 & 0.2 & 1 \\
\hline Computers (desktops/laptops) & 57 purchased & 29 & 0.2 & 1 \\
\hline Meat (canteen) & $1,000 \mathrm{~kg}$ & 16 & 0.1 & $<1$ \\
\hline Total & & $\sim 2,720$ & 18.1 & $100 \%$ \\
\hline
\end{tabular}

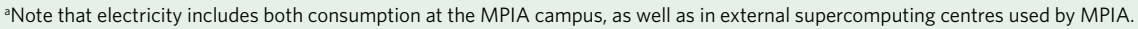

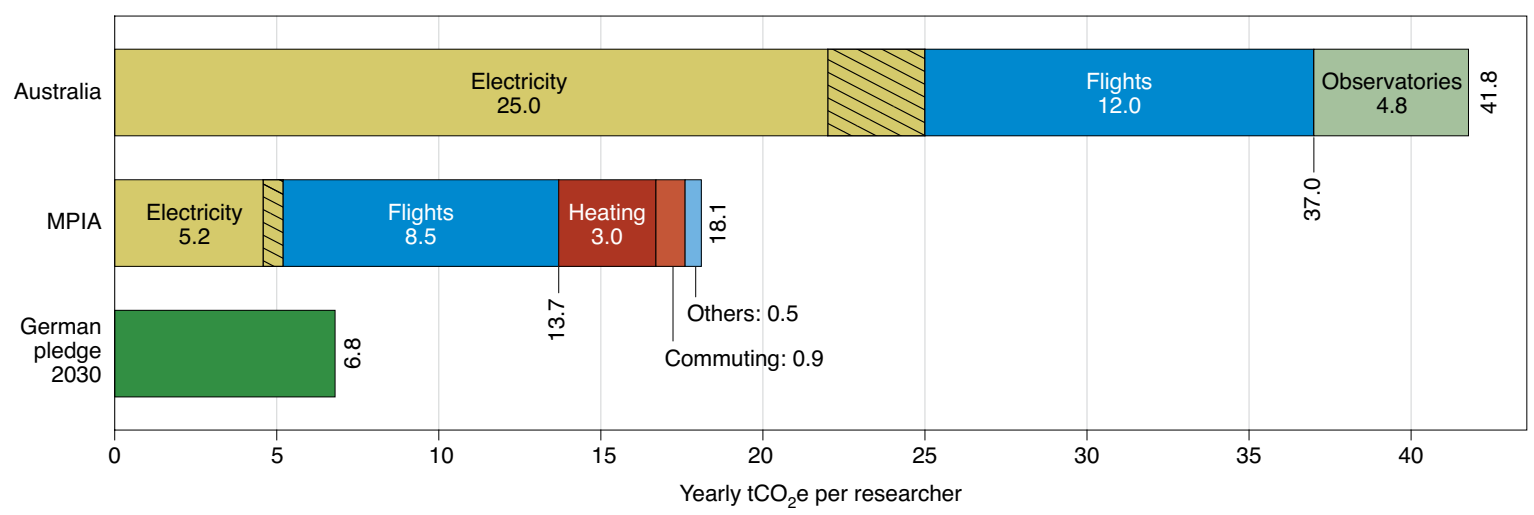

Fig. 1 | Average annual emissions in $\mathbf{2 0 1 8}$ for an Australian and MPIA researcher in $\mathrm{tCO}_{2} \mathrm{e} \mathbf{~ y r}{ }^{-1}$, broken down by sources. The sources include electricity, flights (converted to the same emission model, see text), observatory operation, office heating, commuting and 'others', a category that combines office desktop and laptop hardware, paper and cardboard use, and meat consumption. Electricity-related emissions include both computing and non-computing consumption, and for Australia computing accounts for $88 \%$ of electricity emissions; we estimate a similar fraction for MPIA. In the plot, the smaller hatched part of the 'electricity' bar indicates non-computing electrical power. Observatory operation is only given for Australia, while heating, commuting and sources captured by the 'others' category are only given for MPIA. Therefore, emissions can only be compared between Australia and MPIA for electric power consumption and flights, which amount to 37.0 and $13.7 \mathrm{tCO}_{2} \mathrm{e} \mathrm{yr}^{-1}$ for Australian and MPIA researchers, respectively. The major difference lies in the amount of GHG emissions per kWh electricity, which differs by a factor of around four between Australian astronomy and the MPIA. These values do not account for all emissions per capita. In particular, emissions not related to work are excluded. The combined MPIA emissions of $18.1 \mathrm{tCO}_{2} \mathrm{e} \mathrm{yr}^{-1}$ per researcher are also compared to the German pledge of a 55\% reduction of the 1990 emissions by 2030, plotted per capita in dark green, which is close to $6.8 \mathrm{tCO}{ }_{2}$ e per capita per year ${ }^{9-11}$.

somewhat lower than those of the Australian astronomical community. The second major contributor to the MPIA's GHG emissions is our electricity consumption, at $\sim 5 \mathrm{tCO}_{2} \mathrm{e}$ per astronomer. In contrast, the electricity-related emission, at $22 \mathrm{tCO}_{2} \mathrm{e}$ per astronomer, dominated the Australian astronomers' GHG emissions. The MPIA's electricity consumption mainly results from our computing needs, which for 2018 also included the use of supercomputing facilities in Garching (at the Max Planck Computing and Data Facility), and at the University of Stuttgart for a specific large-scale simulation project. However, the difference to Australia in electricity-related emissions is almost completely due to the different carbon intensity for electricity production: whereas fossil fuel sources contributed $83 \%$ to
Australia's generation of electricity in $2018^{16}$, the contribution in Germany was $\sim 47 \%{ }^{17}$, and MPIA's delivery contracts have a carbon intensity substantially below even that. Thus, for the Australian community, the electricity usage for computing is calculated to require $0.905 \mathrm{~kg} \mathrm{CO}_{2} \mathrm{kWh}^{-1}$, whereas MPIA's electricity contracts average $0.23 \mathrm{~kg}$ $\mathrm{CO}_{2} \mathrm{kWh}^{-1}$. Lastly, we note that the MPIA's heating oil emissions in 2018 are comparable to the 'campus operation' emissions derived for the Australian community (both $3 \mathrm{tCO}_{2} \mathrm{e}$ per researcher), which are extrapolated from the building power requirements of one institute.

\section{Potential measures to reduce emissions}

To reduce our astronomy-related GHG emissions, we need to identify which measures will be effective and require implementation at which level, that is, at the level of the individual researchers, the MPIA, the Max Planck Society, the astronomical community, or human society in general. Each institute will face its specific challenges. For example, we have identified the high carbon intensity of MPIA's heating, which needs to be addressed at the institute level, but other measures need changes across the astronomical community. Measures and responsibilities can only be identified once the GHG emissions have been quantified.

Flying. Flight-related GHG emissions dominate the MPIA's total emissions. Since there is no technology on the horizon that would reduce flight emissions to anything approaching carbon-neutral by 2050 , 


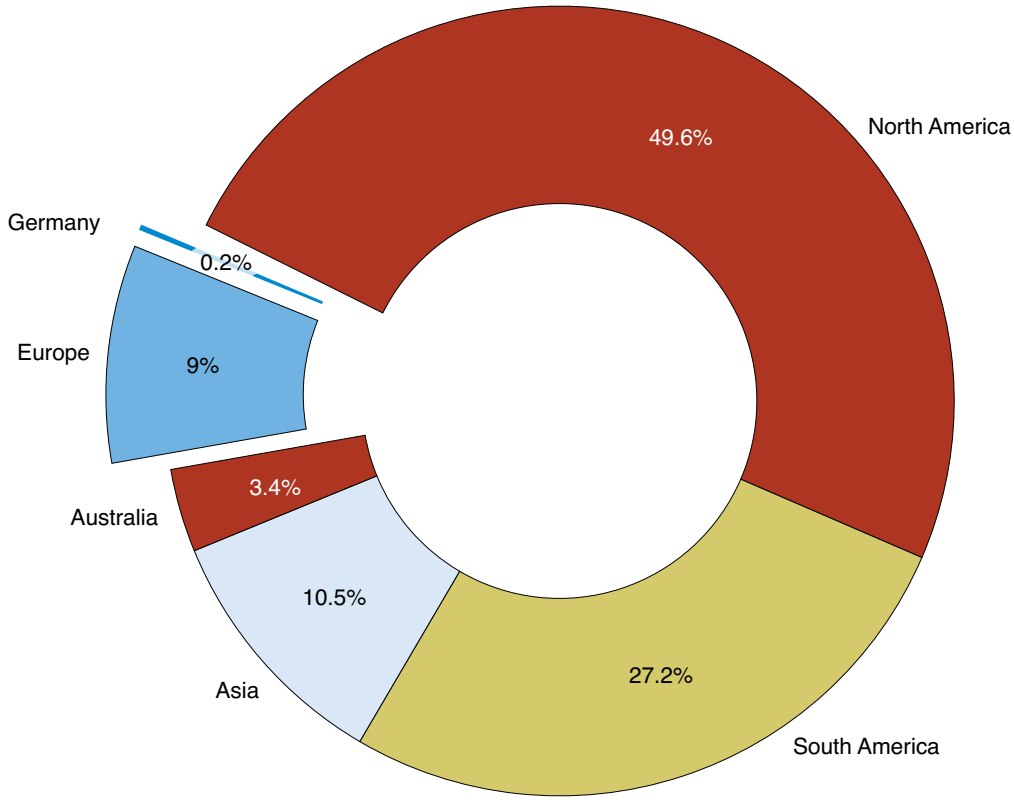

Fig. 2 | Relative GHG emissions broken down by flight destination for MPIA employees.

Intercontinental flights that cannot be easily replaced by alternative means of transport make up about $91 \%$ of flying emissions. This is due to the number of flights, and the high climate impact of each intercontinental flight, primarily due to distance traversed, but also due to greater time-averaged emission altitude, for example, for nitrogen oxides.

much less 2030, the only way to reduce flight-related emissions is to reduce this form of travel. To do so, we need to identify the destinations and reasons for the air travel.

In Fig. 2, we break down the MPIA's emissions by destination. A negligible fraction of emissions originates from flights inside Germany, and only 9\% from flights with destinations inside Europe (including the Canary Islands). Though small, this European component can be further reduced by replacing air travel with train travel ${ }^{7}$. Changes to the German public servant's travel law in early 2020 ensure that train trips to well-connected European destinations are now reimbursed, even if they are more expensive than a flight ${ }^{18}$. Moreover, at the individual level, many German researchers have pledged not to fly distances under $1,000 \mathrm{~km}^{19}$. However, the vast majority of the MPIA's flight emissions (>90\%) stem from intercontinental flights, which are dominated by destinations in the United States and Chile. Although we cannot identify the reason for each flight, in general, these international flights are a mix of travel for observation campaigns, instrument commissioning, conferences, seminars, and research visits. To reduce our flight-related emissions, we must identify solutions that enable us to reach the scientific goals of these trips without the need for air travel. The onus here is on the entire astronomical community to change how we work.

Travel for meetings, conferences and collaborations made up a significant fraction of the MPIA's 2018 flights, as will be the case for most astronomical institutes. At that time, video-based alternatives were only used in specific settings. However, the need to continue working during the 2020 COVID-19 pandemic resulted in the substitution of many physical meetings with virtual ones. To reduce our carbon footprint to anything approaching net zero by 2050 , the expertise in hosting virtual events that was so rapidly developed during the last few months should continue to be applied and expanded. To this end, the recommendations of Klöwer et al. ${ }^{20}$ are an excellent starting point. They provide an in-depth analysis of conferencing carbon emissions and an overview of options. Their analysis shows that GHG emissions for in-person meetings will strongly depend on the meeting location relative to the origin of the participants, and they make cases, for example, for fully online meetings and hybrid models with continental in-person meeting 'hubs', combined with online connections between hubs, as well as other changes that would drastically reduce the conference-induced emissions. These and other models in combination with a drastically lower number of conferences promise to be an effective measure.

In contrast, we identify reasons for flights for which we have no immediate alternatives. These include, for example, extended in-person collaborative visits, which prove very effective for initiating new projects, and the installation or commissioning of instruments at telescopes including the Large Binocular Telescope (Arizona) or the European Southen Observatory Very Large Telescope (Chile). Hardware built by the MPIA must be mounted at a telescope site, tuned, and put into science operations, and as a result, expert engineers and astronomers have to be physically present for a larger number of commissioning runs. Hypothetically, some runs could be combined, but this immediately impacts engineering timescales and family boundary conditions that might be complex to solve. The institute and the astronomy community have to search for measures to address these cases, which at this point are unsolved.

Computing-related emissions. The second major contributor to the MPIA's GHG emissions resulted from the electricity production needed for our computing resources - estimated to be $75-90 \%$ of our electricity consumption - particularly our use of supercomputing facilities. Since large-scale simulations will continue to be an important part of astrophysics in future decades, we need to identify effective measures to reduce the associated emissions. Note that we did not assess the emissions associated with the manufacture of cluster hardware, only of their use.

As is evident from the MPIA/Australian comparison of computing-related emissions, the source of electricity generation has the greatest impact on the computing-related carbon footprint. Thus, it is imperative that supercomputing facilities be run with renewable energy, and that the electricity required for cooling is minimized. The sources of national/regional energy production are decided at a political level, but the astronomical community, and indeed individual citizens, can collectively campaign for this change. As a mid-term option, supercomputing facilities could be moved to locations where renewables are available and less electrical energy is needed for cooling, for example, to Iceland, which had an average of $0.028 \mathrm{~kg} \mathrm{CO}_{2} \mathrm{e} \mathrm{kWh}^{-1}$ emission $^{21}$ for produced electricity in August 2020. Additionally, potential idle times, and hence the required amount of hardware, could be reduced by switching to more cloud computing, because there capacity utilization is generally higher than for local 
computers $^{22}$. As a community, we should guarantee an efficient use of supercomputing resources. This applies to code efficiency ${ }^{23}$ as well as to the computing architecture that we build up or rent ${ }^{24}$. All these options will require changes at the institutional and astronomy-community level.

\section{Heating and local energy production.}

Finally, we briefly touch on the MPIA's buildings. The use of oil for heating at 446 $\mathrm{tCO}_{2} \mathrm{e}$ is the third-largest contributor to the MPIA's GHG emissions. Oil has been used since the institute's buildings were inaugurated in 1976, due to their distance from the city's district heating and gas network. For the future, the only viable and sustainable option for heating the institute is to use ground heat, in combination with an electrically operated heat pump. This type of heating system is already employed at the House of Astronomy, the astronomy education and outreach centre built on the MPIA campus in 2011. Not only can this heating system save $50 \%$ of energy compared to oil-/gas-based systems, but it can also be run carbon-neutral on renewable electricity. Installation of such a heating system can, in principle, be implemented at the institute level, as can improvements to building insulation, which reduce the heating needs. These changes have been proposed for the MPIA and are currently under review.

MPIA's electricity is consumed both on campus for a mix of computing (including cooling), workshops, cleanrooms, and general office consumption, and to a large part in external high-performance computing centres, as described above. While the associated carbon emissions will decrease along with Germany's decreasing use of coal and gas for electricity generation, this process will take a long time. We note that the MPIA's utility contracts have a carbon intensity about half that of the German average, and in principle, for a relatively small extra cost, these contracts could be changed to provide $100 \%$ renewable electricity. However, many such contracts would not actually lead to more renewable energy being produced, but instead only formally redistribute renewable electricity volumes or emission certificates between contracts. Thus, in reality other measures would have a greater impact. We proposed a photovoltaic installation on MPIA's roof, also currently under review, which would initially produce $\sim 10 \%$ of MPIA's on-campus electricity consumption at zero additional cost.

\section{Conclusion}

We have assessed and summarized the MPIA's research-related emissions for the year 2018, finding that the average MPIA astronomer produced at least $18.1 \mathrm{tCO}_{2} \mathrm{e}$ of research-related GHG emissions in that year, a sobering three times the emissions needed for Germany to meet its 2030 goals, set in accordance with the Paris Agreement. We identified the areas in which we produced the most GHG emissions and urge other institutes to conduct their own assessment. Each institute will face a unique set of challenges, depending on its location, funding structure, and fields of research. These challenges can only be addressed once quantified $^{25}$. However, many of the challenges will overlap, as is apparent from our comparison of the MPIA's emissions to those of the Australian astronomical community.

We identified a high carbon cost associated with astronomy-wide issues, but also a few that were institute-specific. Overall, work-related travel dominates our carbon footprint and must be addressed as a community. If we continue to travel by air as we do now, we will not meet the required global reduction in $\mathrm{CO}_{2}$ emissions. The astronomical community should adopt some of the recommendations of Klöwer et al..$^{20}$, and go beyond them in some respects. The second dominant contribution is the electricity generation for computations on clusters. Changes in the production of electricity are required to address this in the long term, but we can start to partially address this at the institute level with on-site, renewable means of energy production. For example, we have proposed the installation of solar panels on the flat and vacant MPIA roof space. The third highest contribution, which was institute-specific, was the high carbon footprint associated with heating. We have recommended that the heating system be changed to a ground-heating system in the future.

We require both a local and community-wide approach to reduce the GHG emissions associated with astronomy research. For this, we need the lead of both our professional organizations (for example, the International Astronomical Union, and the European and American astronomical societies) and funding agencies, as well as the development and leading by example of larger institutes or communities. The political landscape is unlikely to adapt rapidly enough to the evolving climate crisis situation. Instead, we as astronomers need to 'own' our emissions and adapt the culture and technology we use to conduct our research. In doing so, we can set an example for others to follow.

\section{Knud Jahnke (D) 1,2四, Christian Fendt ${ }^{1,2}$, Morgan Fouesneau ${ }^{1,2}$, Iskren Georgiev ${ }^{1,2}$,}

Tom Herbst ${ }^{1,2}$, Melanie Kaasinen ${ }^{1,2}$, Diana Kossakowski (D) 1,2, Jan Rybizki, ${ }^{1,2}$ Martin Schlecker (D) 1,2, Gregor Seidel ${ }^{1,2}$, Thomas Henning (D)', Laura Kreidberg ${ }^{1}$ and Hans-Walter Rix

${ }^{1}$ Max Planck Institute for Astronomy, Heidelberg,

Germany. ${ }^{2}$ These authors contributed equally:

Knud Jahnke, Christian Fendt, Morgan Fouesneau, Iskren Georgiev, Tom Herbst, Melanie Kaasinen, Diana Kossakowski, Jan Rybizki, Martin Schlecker, Gregor Seidel.

$凶_{e-m a i l: j a h n k e @ m p i a . d e}$

Published online: 10 September 2020

https://doi.org/10.1038/s41550-020-1202-4

References

1. McMichael, A. J. et al. Climate Change and Human Health: Risks and Responses (World Health Organization, 2003).

2. Edenhofer, O. et al. IPCC, 2014: Summary for Policymakers (IPCC, 2014).

3. Rohde, R. Global Temperature Report for 2019 (Berkeley Earth, 2019); https://berkeleyearth.org/2019-temperatures-new/

4. The Paris Agreement (United Nations, 2016); https://unfccc.int/ process-and-meetings/the-paris-agreement/the-paris-agreement

5. Stevens, A. R. H., Bellstedt, S., Elahi, P. J. \& Murphy, M. T. Nat. Astron. https://doi.org/10.1038/s41550-020-1169-1 (2020).

6. Flagey, N. \& Thronas, K., Petric, A., Withington, K. \& Seidel, M. J. Nat. Astron. https://doi.org/10.1038/s41550-020-1190-4 (2020).

7. Burtscher, L. et al. Nat. Astron. https://doi.org/10.1038/s41550020-1207-z (2020)

8. Marshall, P. J. et al. Preprint at https://arxiv.org/abs/0903.3384 (2009).

9. Submission Under the United Nations Framework Convention on Climate Change and the Kyoto Protocol 2020: National Inventory Report for the German Greenhouse Gas Inventory 1990-2018 (Federal Environment Agency, 2020); https://go.nature. com/2PQCje0.

10. Federal Climate Protection Act (BMU, 2019); https://www.bmu.de/ gesetz/bundes-klimaschutzgesetz

11. Recalculated and Extrapolated Population Based on the 2011-1991 to 2011 Census (Destatis, 2016); https://go.nature.com/3iBuuF9

12. Germany Current Population (Destatis, 2020); https://go.nature. $\mathrm{com} / 2 \mathrm{FcKleW}$

13. Ritchie, H. \& Roser, $\mathrm{M} . \mathrm{CO}_{2}$ and Greenhouse Gas Emissions (Our World in Data, 2017); https://ourworldindata.org/ co2-and-other-greenhouse-gas-emissions

14. atmosfair Emissions Calculator (atmosfair $\mathrm{gGmbH}$, accessed 24 July 2020); https://www.atmosfair.de/en/standards/ emissions_calculation/emissions_calculator

15. Qantas Future Planet (Qantas, accessed 24 July 2020); https:// www.qantasfutureplanet.com.au

16. Table O: Australian Electricity Generation, By Fuel Type, Physical Units (Department of the Environment and Energy, 2019); https:// www.energy.gov.au/publications/australian-energy-update-2019

17. Net Public Electricity Generation in Germany in 2018 (Fraunhofer ISE, accessed 24 July 2020); https://www.energy-charts.de/ energy_pie.htm?year $=2018$

18. Contribution to Climate Protection: More Business Trips by Train (BMI, 28 January 2020); https://www.bmi.bund.de/SharedDocs/ kurzmeldungen/DE/2020/01/brkg-bahn.html

19. Voluntary Commitment to Refrain from Short-haul Business Flights (Scientists for Future, 2020); https://unter1000.scientists4future.org

20. Klöwer, M., Hopkins, D., Allen, M. \& Higham, J. Nature 583, 356-359 (2020)

21. electricityMap (Tomorrow, accessed 3 August 2020); https://www. electricitymap.org/zone/IS

22. Radu, L.-D. Symmetry 9, 295 (2017)

23. Portegies Zwart, S. Nat. Astron. https://doi.org/10.1038/s41550020-1208-y (2020).

24. Henderson, P. et al. Preprint at https://arxiv.org/abs/2002.05651 (2020).

25. Matzner, C. et al. Astronomy in a Low-Carbon Future (Zenodo, 2019); https://zenodo.org/record/3758549

Competing interests

The authors declare no competing interests. 